\title{
Estudio de la susceptibilidad de un acero inoxidable dúplex del tipo 22Cr5NiMoN al dañado por hidrógeno en condiciones estáticas (HIC) y bajo carga (SSC)
}

\author{
S. Gutiérrez de Saiz-Solabarría ${ }^{(*)}$, J.M. San Juan ${ }^{(* *)}$ y A. Valea ${ }^{(* * *)}$
}

\begin{abstract}
Resumen Se estudia el comportamiento de un mismo acero moldeado inoxidable dúplex austeno-ferrítico (48$52 \%$ ) del tipo 22Cr5NiMoN (UNS-J9.22.05) frente al dañado por hidrógeno generado por corrosión en medio $\mathrm{H}_{2} \mathrm{~S}$, tanto en condiciones estáticas, mecanismo de dañado conocido como HIC (Hydrogen Induced Cracking), como bajo carga de tracción, mecanismo de dañado conocido como SSC (Sulfide Stress Cracking).
\end{abstract}

Palabras clave: Acero moldeado dúplex 22Cr5NiMoN. Dañado por hidrógeno (HIC). Corrosión bajo tensión en medio sulfhídrico (SSC).

\section{Study of the susceptibility of a 22Cr5NiMoN-type duplex stainless steel to hydrogen damage under static conditions (HIC) and under sustained load (SSC)}

Abstract The behavior to hydrogen damage caused by corrosion in a $\mathrm{H}_{2} \mathrm{~S}$ medium is studied in a molded ferri-
te-austenite (52-48 \%) duplex stainless steel $22 \mathrm{Cr} 5 \mathrm{NiMoN}$ type (UNS-J9.22.05) under both, static
(damaging mechanism called Hydrogen Induced Cracking (HIC)) and sustained load (damaging mechanism called Sulfide Stress Cracking (SSC)), conditions.

Keywords: Molded 22Cr5NiMoN duplex stainless steel. Hydrogen induced cracking (HIC). Sulfide stress cracking (SSC).

\section{INTRODUCCIÓN}

Se estudia el comportamiento de un acero inoxidable dúplex austeno-ferrítico con una fracción de volumen de ferrita del $52 \%$ frente al dañado por hidrógeno generado durante la corrosión en medio $\mathrm{H}_{2} \mathrm{~S}$, tanto en condiciones estáticas o sin esfuerzo aplicado (Hydrogen Induced Cracking, HIC) como

(*) Dpto. Laboratorios. Unidad I+D. Babcock \& Wilcox Spain S.A. BWE/SEPI. Aptdo. 294. 48080-Bilbao y Dpto. Ciencia Materiales e Ing. Metalúrgica. Univ. del País Vasco. UPV/EHU. Aptdo. 644. 48012-Bilbao (España).

(**) Dpto. Física de la Materia Condensada. Metalurgia Física. Fac. de Ciencias. Campus de Leioa. Univ. del País Vasco. UPV/EHU. Aptdo. 644. 48080-Bilbao (España).

(***) Dpto. Ingeniería Química y del Medio Ambiente. E. Univ. de Ingeniería T. Industrial. Univ. del País Vasco. UPV/EHU. Aptdo. 644. 48012-Bilbao (España). bajo carga de tracción uniaxial (Sulfide Stress Cracking, SSC). La reacción global de corrosión que tiene lugar es: $\mathrm{H}_{2} \mathrm{~S}+\mathrm{Fe}$ (del acero) $\rightarrow \mathrm{FeS}+\mathrm{H}^{0}$ (atómico). A continuación, el hidrógeno generado en la superficie metálica se absorbe y difunde a través del material mediante mecanismos cuasificknianos y se recombina para formar hidrógeno molecular en trampas tales como inclusiones $(\mathrm{MnS})$, precipitados, partículas de segunda fase, cavidades. De este modo, se alcanzan altas presiones locales de $\mathrm{H}_{2}$ que dan lugar a la fractura del material (1-4).

\section{MATERIALES}

El material utilizado es un acero inoxidable dúplex austeno-ferrítico del tipo UNS-J9.22.05 (ACI$\mathrm{CD}-3 \mathrm{MN}$ ) moldeado, que cumple con el documento 
TABLA I.- Composición química del material estudiado (Índice PREN $=\mathrm{Cr}+3,3 \mathrm{Mo}+16 \mathrm{~N}$ )

TABLE I.- Chemical composition of the studied material $(P R E N=C r+3,3$ Mo y $16 \mathrm{~N})$

\begin{tabular}{|c|c|c|c|c|c|c|c|c|c|c|c|}
\hline \multirow[b]{2}{*}{ Material } & \multicolumn{11}{|c|}{ Composición, $\%$ en masa } \\
\hline & $\mathrm{C}$ & Mn & $\mathrm{Si}$ & $\mathrm{P}$ & $S$ & $\mathrm{Cr}$ & $\mathrm{Ni}$ & Mo & $\mathrm{Cu}$ & $\begin{array}{c}\mathrm{N} \\
\text { (total) }\end{array}$ & PREN \\
\hline $\begin{array}{l}\text { 019C-A/B } \\
\text { (Probetas) }\end{array}$ & 0,028 & 0,65 & 0,70 & 0,020 & 0,001 & 22,78 & 5,57 & 3,38 & 0,11 & 0,16 & 36,5 \\
\hline $\begin{array}{c}\text { Según ASTM } \\
\text { A890 Gr4A } \\
(22 \mathrm{Cr} 5 \mathrm{NiMoN})\end{array}$ & $\begin{array}{c}\leq \\
0,03\end{array}$ & $\begin{array}{l}\leq \\
1,50\end{array}$ & $\begin{array}{l}\leq \\
1,00\end{array}$ & $\begin{array}{c}\leq \\
0,040\end{array}$ & $\begin{array}{c}\leq \\
0,020\end{array}$ & $\begin{array}{c}21,0 \\
\div \\
23,5\end{array}$ & $\begin{array}{c}4,5 \\
\div \\
6,5\end{array}$ & $\begin{array}{c}2,5 \\
\div \\
3,5\end{array}$ & $\begin{array}{l}\leq \\
1,00\end{array}$ & $\begin{array}{c}0,10 \\
\div \\
0,20 \\
\end{array}$ & $\begin{array}{c}31,0 \\
\div \\
38,0 \\
\end{array}$ \\
\hline
\end{tabular}

estándar A890-94 de la norma ASTM (5) para su grado Gr 4A (22Cr5NiMoN). La composición química determinada por técnicas analíticas instrumentales de espectrometría de emisión atómica por descarga eléctrica en alto vacío (AES) y por plasma de acoplamiento inductivo (ICP), se muestra en la tabla I. El estudio de la microestructura puso de manifiesto la presencia de dos fases: austenita-ferrita, en una fracción de volumen del 48 y $52 \%$, respectivamente (Fig. 1). Así mismo, se observó la presencia de carburos preferentemente en borde de gano austenítico (Fig. 2). La dureza es de $98 \mathrm{HRB}$, que se corresponde con una resistencia a la tracción del orden de $80 \mathrm{~kg} / \mathrm{mm}^{2}$.

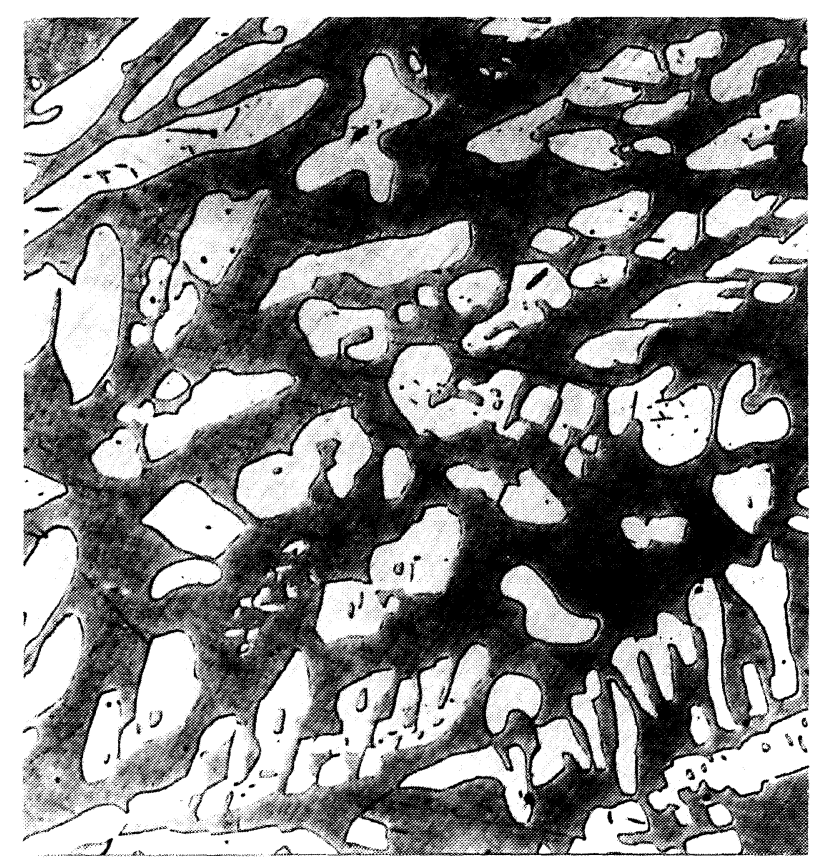

FIG. 1.- Acero moldeado duplex 22Cr5NiMoN. Austenita-Ferrita (48-52\%). (× 100).

FIG. 1.-22Cr5NiMoN duplex molded steel. Austenite-Ferrite $(48-52 \%) .(\times 100)$.

\section{PARTE EXPERIMENTAL}

\subsection{Dañado por hidrógeno (HIC)}

La metodología experimental que se aplica para evaluar el dañado por hidrógeno se corresponde con lo expuesto por NACE en su documento normalizado de referencia TM.02.84-87 (6).

\subsubsection{Probetas}

Se mecanizaron dos probetas prismáticas de 100 $\pm 1 \mathrm{~mm}$ de longitud, $20 \pm 1 \mathrm{~mm}$ de anchura y $15 \pm$ $1 \mathrm{~mm}$, haciendo coincidir la dirección longitudinal

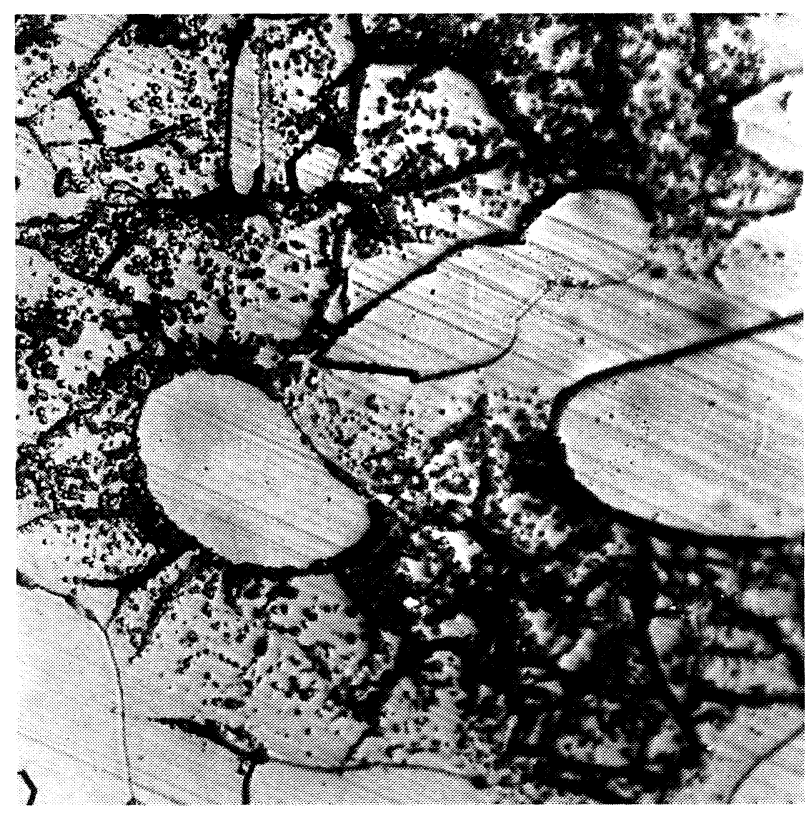

FIG. 2.- Acero moldeado duples $22 \mathrm{Cr} 5 \mathrm{NiMoN}$. Austenita-Ferrita. Precipitación de carburos. $(\times 500)$.

FIG. 2.-22Cr5NiMoN duplex molded steel. Austenite-Ferrite. Carbides precipitation. $(\times 500)$. 
de la probeta con la dirección de solidificación. Las seis caras de la probeta se lijaron y pulieron hasta grano 320 ó más fino. A continuación, las seis caras se limpiaron con acetona o similar.

\subsubsection{Solución de ensayo}

Se utilizó agua de mar sintética purgada con nitrógeno y saturada de sulfuro de hidrógeno $\left(\mathrm{H}_{2} \mathrm{~S}\right)$. El agua de mar sintética se prepara cumpliendo lo expuesto por ASTM en el documento normalizado de referencia D 1141-90 (7). El agua utilizada para disolver las diferentes sales es de grado reactivo tipo II de acuerdo con ASTM D1193 (8) y la solución $0,1 \mathrm{~N}$ de $\mathrm{NaOH}$ utilizada para ajustar el $\mathrm{pH}$ cumple con ASTM E200 (9). El agua de mar sintética que se utilizó tiene la siguiente composición, en g/L: $24,53 \mathrm{NaCl}+5,20 \mathrm{MgCl}_{2}+4,09 \mathrm{Na}_{2} \mathrm{SO}_{4}+1,16$ $\mathrm{CaCl}_{2}+0,695 \mathrm{KCl}+0,201 \mathrm{NaHCO}_{3}+0,101 \mathrm{KBr}+$ $0,027 \mathrm{H}_{3} \mathrm{BO}_{3}+0,025 \mathrm{SrCl}_{2}+0,003 \mathrm{NaF}$. Presenta, por tanto, un índice de clorabilidad de 19,38 y un $\mathrm{pH}$ de 8,2 después de ajustar con $\mathrm{NaOH} 0,1 \mathrm{~N}$.

\subsubsection{Purga y saturación}

El aire disuelto en la solución de agua de mar sintética se eliminó haciendo circular un caudal de nitrógeno de $100 \mathrm{~cm}^{3} / \mathrm{min}(6 \mathrm{~L} / \mathrm{h})$ por litro de solución. A continuación se saturó la solución con un caudal de $\mathrm{H}_{2} \mathrm{~S}$ de $200 \mathrm{~cm}^{3} / \mathrm{min}(12 \mathrm{~L} / \mathrm{h})$ por litro de solución, durante $1 \mathrm{~h}$. Para asegurar la saturación de $\mathrm{H}_{2} \mathrm{~S}$ durante las $96 \mathrm{~h}$ de ensayo, se mantuvo un burbujeo de dicho gas de $10 \mathrm{~cm}^{3} / \mathrm{min}(0,6 \mathrm{~L} / \mathrm{h})$ y por litro de disolución.

\subsubsection{Parámetros de ensayo}

Según lo expuesto en las normas usadas, el $\mathrm{pH}$ del agua de mar sintética, inicialmente preparada, estará entre 8,1 y 8,3 (en nuestro caso, $\mathrm{pH}=8,2$ ) y al finalizar el ensayo estará entre 4,8 y 5,4 (en nuestro caso, $\mathrm{pH}=5,0$ ). El ensayo se realizó bajo presión atmosférica a $25 \pm 2{ }^{\circ} \mathrm{C}$ durante $96 \mathrm{~h}$.

\subsubsection{Evaluación}

Después de permanecer $96 \mathrm{~h}$ inmersas en la solución de ensayo, se sacaron las probetas del reactor, se limpiaron y secaron. A continuación, se procedió a la evaluación macroscópica de las superficies expuestas para detectar la presencia de ampollas (blistering). Posteriormente, las probetas se seccionaron transversalmente, cada $25 \mathrm{~mm}$. Las superficies obtenidas se lijaron, pulieron metalográ- ficamente y atacaron ligeramente con un reactivo específico, a fin de evaluar la presencia de grietas. Se evaluaron los siguientes parámetros de dañado: a) tamaño, localización y morfología de la ampolla; b) relación de longitud de agrietamiento $(C L R=\Sigma$ $a / w)$, anchura del agrietamiento $(C T R=\Sigma b / w)$ y sensibilidad al agrietamiento $(C S R=\Sigma(a \cdot b) /(w$. $T)$ ), donde $a$ es la longitud y $b$ la anchura de cada grieta, $w$, la anchura y $T$ el espesor de la probeta, y e) morfología de la grieta.

\subsection{Corrosión bajo tensión en medio sulfhídrico (SSC)}

La metodología experimental aplicada para evaluar el dañado por hidrógeno se ajusta a lo expuesto por NACE en su documento normalizado de referencia TM.01.77-90 (10).

\subsubsection{Probetas}

Se mecanizaron dos probetas de tracción cilíndricas de cabezas roscadas haciendo coincidir la dirección longitudinal de la probeta con la dirección de solidificación. Su configuración geométrica y sus dimensiones se muestran en la figura 3. Se hizo un acabado final de la probeta hasta una rugosidad de $0,81 \mu \mathrm{m}$ o inferior. Antes del ensayo, la probeta se desengrasó con 1,1,1 tricloroetano o similar y se lavó con acetona.

\subsubsection{Solución de ensayo}

Se utilizó una solución acuosa acidificada saturada de $\mathrm{H}_{2} \mathrm{~S}$. La acidificación se realizó preparando $50,0 \mathrm{~g}$ de $\mathrm{NaCl}+5,0 \mathrm{~g}$ de ácido acético glacial en $945 \mathrm{~g}$ de agua destilada $(5,0 \%$ en masa de $\mathrm{NaCl}$ y $0,5 \%$ en peso de ácido acético glacial). Al igual que en los ensayos de dañado por hidrógeno, se eliminó el aire disuelto en la disolución con un caudal de nitrógeno y se saturó con $\mathrm{H}_{2} \mathrm{~S}$. El NaCl y el ácido acético son de Merck y de grado reactivo (pureza $\cong 99,5$ $\%)$. La calidad del agua destilada utilizada cumple con lo expuesto en el documento normalizado D 1193 de ASTM (8). La relación volumen de solución/superficie de la probeta es de $30 \pm 5 \mathrm{~mL} / \mathrm{cm}^{2}$.

\subsubsection{Parámetros de ensayo}

La solución de ensayo preparada, como se ha comentado anteriormente, presenta un $\mathrm{pH}=3,0$. Durante el ensayo, el $\mathrm{pH}$ se incrementa a $\mathrm{pH}=4,0$. El ensayo se realizó bajo la presión atmósférica durante $720 \mathrm{~h}$ (si la probeta no rompe antes). Durante el ensayo, la probeta permaneció inmersa en la 


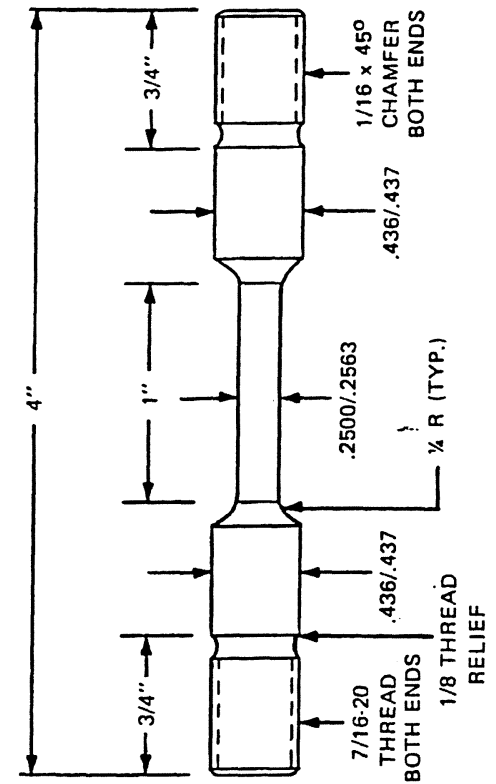

FIG. 3.- Probeta para estudio del SSC.

$$
\text { FIG. 3.- Samples of SSC study. }
$$

solución de ensayo, que se mantuvo a una temperatura de $25 \pm 2{ }^{\circ} \mathrm{C}$.

\subsubsection{Secuencia de experimentación}

La probeta de tracción se montó con sus extremos roscados acoplados a un anillo dinamométrico, mientras que el resto de la probeta permaneció inmersa en la solución de ensayo. A continuación, se cargó la probeta uniaxialmente en tracción, flexionando el anillo dinamométrico con un dispositivo al efecto. El desplazamiento por flexión del anillo está relacionado linealmente con la carga aplicada, que corresponde a un determinado porcentaje del valor de la tensión del límite elástico del material investigado (en nuestro caso, $80 \%$ ). A continuación, y con el reactor sellado, se purgó la disolución con un caudal de nitrógeno de $100 \mathrm{~mL} / \mathrm{min}(6 \mathrm{~L} / \mathrm{h})$ durante $1 \mathrm{~h} / \mathrm{L}$ de solución. Seguidamente, se saturó con un caudal de $\mathrm{H}_{2} \mathrm{~S}$ de $200 \mathrm{~mL} / \mathrm{min}(12 \mathrm{~L} / \mathrm{h})$ durante 1 h/L de solución. El mantenimiento de la saturación durante todo el ensayo se aseguró mediante burbujeo de $\mathrm{H}_{2} \mathrm{~S}$ a razón de $10 \mathrm{~mL} / \mathrm{min}(0,6 \mathrm{~L} / \mathrm{h})$.

\subsubsection{Evaluación}

Para la medición del tiempo de rotura, se dispuso de unos microsensores de limitación de recorrido en contacto con el anillo dinamométrico. Si transcurridas las $720 \mathrm{~h}$ de ensayo, la probeta no rompe, se evalúa la presencia de grietas, fisura o cualquier tipo de anomalía presente sobre la superficie de la probeta.

\section{RESULTADOS}

\subsection{Dañado por hidrógeno (HIC)}

La superficie de las probetas ensayadas no presentó ni ampollas (blisters), ni grietas, como se observa tanto en las superficies laterales (Fig. 4), como en las secciones metalográficas transversales (Fig. 5). Por esta razón, se deduce que los parámetros $C L R, C T R$ y $C S R$ son $0,00 \%$.

\subsection{Corrosión bajo tensión en medio sulfhídrico (SSC)}

Las dos probetas ensayadas rompieron a las 82 y $99 \mathrm{~h}$ de ensayo, respectivamente. La superficie de fractura es casi perpendicular a la dirección de tracción, como se observa en la figura 6 . En la figura 7 se presenta un detalle de una de las superficies de fractura.

\section{CONCLUSIONES}

- El acero dúplex 22Cr5NiMoN moldeado no es susceptible al daño por hidrógeno (HIC).

- El acero dúplex 22Cr5NiMoN moldeado es susceptible a la corrosión bajo tensión en medio sulfhídrico (SSC) cuando se somete en tracción a una carga correspondiente al $80 \%$ del valor de su límite elástico. La superficie de fractura de la probeta es prácticamente perpendicular a la dirección de tracción. La fractura del material se asocia a la gran cantidad de carburos presentes en la microestructura.

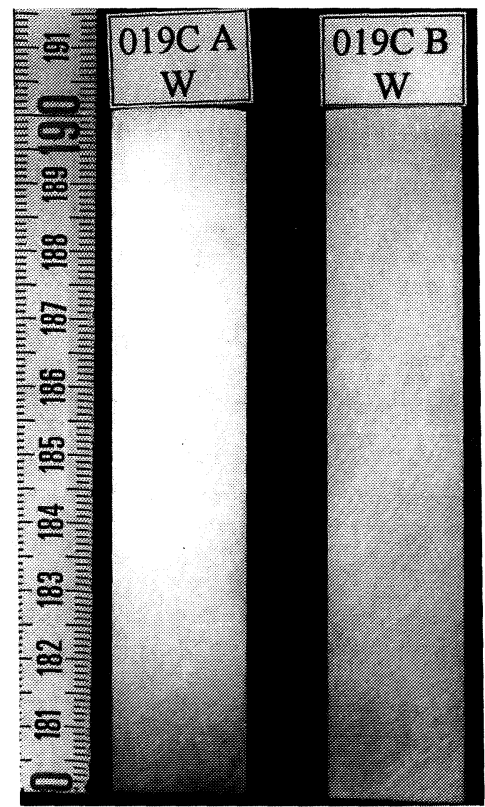

FIG. 4.- HIC. Probetas después del ensayo. $(\times 1)$

FIG. 4.- HIC. Samples after test $(\times 1)$. 


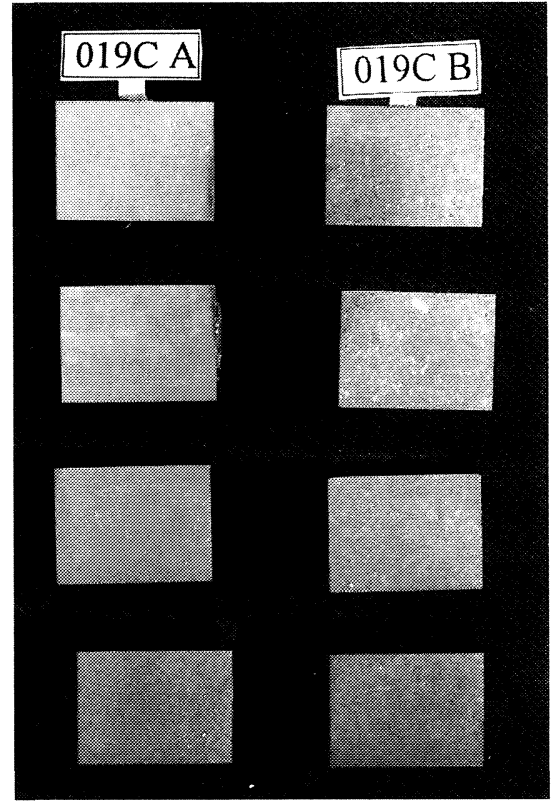

FIG. 5.- HIC. Secciones metalográficas transversales. $(\times 1)$.

FIG. 5.- HIC. Metallographical cross sections. $(\times 1)$.

\section{REFERENCIAS}

(1) Gutiérrez, S., Valea, A. y González, M.L. Estudio de la susceptibilidad al agrietamiento por HIC en materiales metálicos del tipo API 5L Gr X5L, X60 y X65. XXIII Bienal de la RSEQ. Salamanca (España). 1990.

(2) Gutiérrez, S. y ValeA, A. Estudio de la susceptibilidad al agrietamiento por SSC en materiales metálicos del tipo API 5L Gr X60 y X65. XXIV Bienal de la RSEQ. Málaga (España). 1992.

(3) GutiérREZ, S. Evaluación del comportamiento de materiales metálicos sometidos a ambientes de $\mathrm{H}_{2} \mathrm{~S}$ en condiciones estáticas y dinámicas. X Cong. Nac. de Ing. Mecánica. Madrid (España). 1992.

(4) Gutiérrez, S., San Juan, J.M. y Valea, A. Estudio de la influencia del $\mathrm{pH}$ sobre el dañado por HIC en aceros CMn microaleados con V y Nb. XXVI Bienal de la RSEQ. Cádiz (España). 1997.

(5) ASTM A 890/890M-94a. Standard Specification for Castings, Iron-Chromium-Nickel-Molybdenum Corrosion Resistant, Duplex for General Application. ASTM. Section I. Volume 01-02. pp. 595-597. West Conshohocken. (PA, EE.UU.) 1997.

(6) NACE TM.02.84-87. Standard Test Method. Evaluation of Pipeline Steels for Resistance to Stepwise Cracking. NACE. Aprobado Feb. 1984. Reafirmado Mar. 1987. Revisado 1996, Mar. 30. Houston (TX, EE.UU.) 1987.

(7) ASTM D1141-90. Standard Specification for Substitute Ocean Water. ASTM Standards for Corrosion Testing of Metals. pp. 132-133. ASTM. Filadelfia (PA, EE.UU.) 1990.

(8) ASTM D1193-77 (Reapproved 1983). Standard Specification for Reagent Water. ASTM Standards for Corrosion

\author{
PROBETAS DESPUÉS DEL ENSAYO \\ 019C - A $\quad 019 \mathrm{C}-\mathrm{B}$ \\ (rotura $t=82 \mathrm{~h}) \quad($ rotura $t=99 \mathrm{~h})$
}

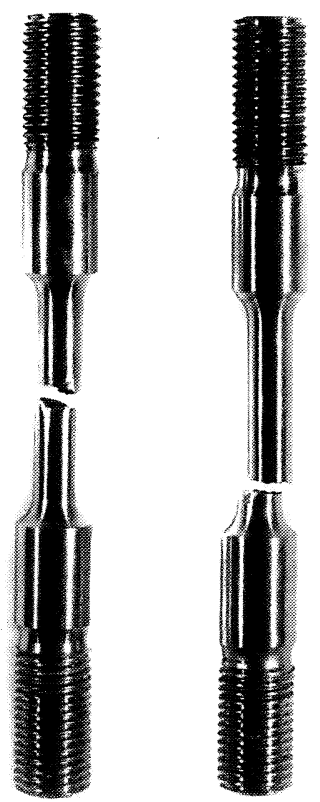

FIG. 6. - SSC. Probetas con rotura total. $(\times 1)$.

FIG. 6. - SSC. Samples with total cracking. $(\times 1)$.

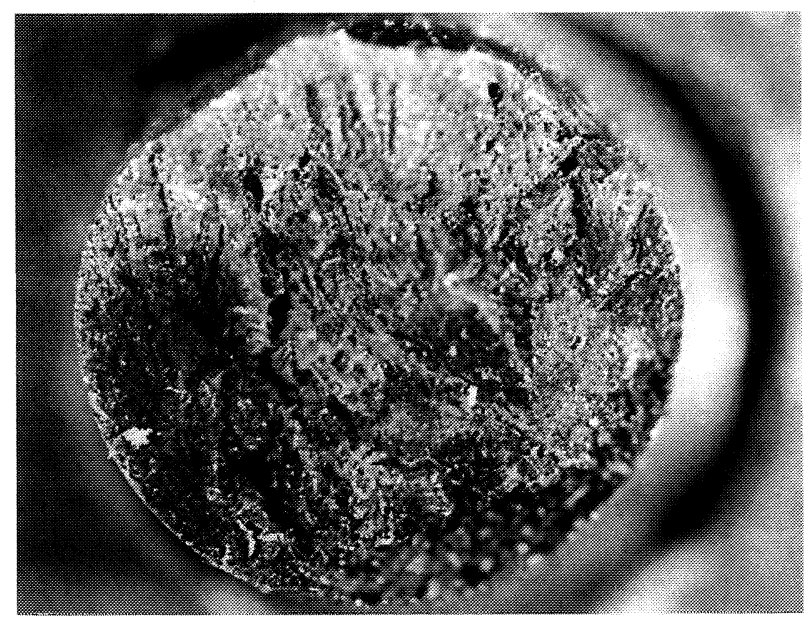

FIG. 7.- SSC. Superficie de fractura. $(\times 14)$.

FIG. 7.-SSC. Fracture Surface. $(\times 14)$.

Testing of Metals. pp. 134-136. ASTM. Filadelfia, (PA, EE.UU.) 1990.

(9) ASTM E200-86. Practice for Preparation, Standarization and Storage of Standard Solutions for Chemical Analysis. ASTM. Vol 11.01 Filadelfia PA. (EE.UU.) 1991.

(10) NACE TM.01.77-90. Standard Test Method. Laboratory Testing of Metals for Resistance to SSC in $\mathrm{H}_{2} \mathrm{~S}$ Environment. NACE. Aprobado Jul. 1977. Revisado Ene. 1986. Revisado Mar. 1990. Revisado 1996, Dic. 23. Houston (TX, EE.UU.) 1990. 